\title{
Arabic Diacritics Based Steganography
}

Mohammed A. Aabed, Sameh M. Awaideh, Abdul-Rahman M. Elshafei and Adnan A. Gutub

\author{
King Fahd University of Petroleum and Minerals \\ Computer Engineering Department \\ Dhahran 31261, Saudi Arabia \\ \{maabed, sameho, shafei, gutub\}@kfupm.edu.sa
}

\begin{abstract}
New steganography methods are being proposed to embed secret information into text cover media in order to search for new possibilities employing languages other than English. This paper utilizes the advantages of diacritics in Arabic to implement text steganography. Diacritics - or Harakat - in Arabic are used to represent vowel sounds and can be found in many formal and religious documents. The proposed approach uses eight different diacritical symbols in Arabic to hide binary bits in the original cover media. The embedded data are then extracted by reading the diacritics from the document and translating them back to binary.
\end{abstract}

Index Terms - Arabic Text, Data Hiding, Diacritic Marks, Text Steganography, Text Watermarking

\section{Introduction}

The evolution experienced nowadays in computer technologies is employing a rapid growth in the number of users and diversity of applications. One of the main concerns in this field is the ability to privately exchange information and hide the data of interest throughout the transmission process. A variety of solutions have been proposed for this problem in the literature, some are cryptographic, steganographic or even pure coding. As a way to hide the exchange of data, steganography has gained a wide interest among researchers and security specialists [10].

Steganography is the science of forming hidden messages such that the intended recipient is the only party aware of the existence of the message [1]. This is usually done by embedding the private data in a cover media without destroying the meaningfulness of this media. The fact that people are not aware of the presence of the message distinguishes between steganography and other forms of information security. For instance, in cryptographic systems the existence of the information itself is not disguised although the comprehension of the message is not possible [2].

In steganography for digital systems, the cover media used to hide the message can be text, image, video or audio files [3]. However, using text media for this purpose is considered the hardest among the other kinds. Unlike video and voice media, text data does not have much needless information within the essential data $[4,5]$.

Different methods and approaches have been attempted to implement text steganography [4]. Most of these known approaches hide data by making minimal modifications to the painting of the characters or spaces. The authors in [4] name feature coding, open spaces, word shifting and line shifting as examples for the these 
approaches. It should be noted that these approaches are usually hard to implement and are font or computer dependent. Other approaches use syntactic and semantic characteristics in the language to embed data. Additional implemented methods for text steganography using Arabic language are further discussed in the following sections.

This paper proposes a new steganography scheme to hide binary data in Arabic text media. The rest of this paper is organized as follows; section 2 has the background information about Standard Arabic language and related work. In section 3, the new approach is introduced in details. The analysis and discussion are reported in section 4. Finally, the conclusion statement is in section 5.

\section{Background}

The Arabic language, written from right to left, is based on an alphabetical system that uses 28 basic letters. Unlike English, Arabic does not differentiate between upper and lower case or between written and printed letters. Moreover, Arabic language uses different symbols as diacritical marks, or simply diacritics which are also known as Harakat. The main eight diacritic symbols are shown in Table 1. Other diacritic marks also exist but are outside of the scope of this paper.

Table 1. The eight main Arabic diacritics

\begin{tabular}{|c|c|c|c|}
\hline Fatha & $=$ & Kasrah & $=$ \\
\hline Dhammah & $\underline{-}$ & Sukkon & - \\
\hline Shaddah & $\simeq$ & Tanween Fath & $\equiv$ \\
\hline Tanween Kasr & $\bar{z}$ & Tanween Dham & $\underline{*}$ \\
\hline
\end{tabular}

Just like most of the diacritics based written languages, the main purpose of using diacritics in Arabic languages is to alter the pronunciation of a phoneme or to distinguish between words of similar spelling. Nonetheless, the use of diacritics in the text is optional in written Standard Arabic. In this work we utilize this characteristic to define a steganographic scheme for hiding binary data within Arabic text, as it will be shown in details in section 3 .

Arabic language is poorly used in the steganography field. To the best of the authors' knowledge, there is only one algorithm in the literature that uses Arabic text as a cover media to hide binary data [4]. The authors in [4] proposed a feature coding method for hiding binary values into Persian/Arabic phrases. This method takes advantage of the frequent presence of point in these kinds of phrases. Simply, the approach uses vertical repositioning of these points to represent 0 's and 1's. The paper reports some of the advantages and disadvantages of the proposed algorithm and discusses difficulties facing text steganography as well.

Another proposed - yet unpublished - approach exploits the existence of the redundant Arabic extension character, i.e. Kashida [6, 11]. The author proposes to use pointed letters in Arabic with a Kashida to hold the secret bit 'one' and the un-pointed letters with a Kashida to hold 'zero'. This approach as well as other approaches will be used for analysis and comparison of the results. Before embarking upon our proposed approach, it should be noted that this paper is not restricted only for the Arabic language. Most of the Semitic languages use diacritics in one form or another, 
and the proposed approach can be slightly modified to suit other languages requirements.

\section{Proposed Approach}

The use of diacritics in written Standard Arabic language is optional. This means that novel Arabic readers can read a text without diacritics correctly by applying the Arabic language grammar to that text. In this work, we use this property to introduce a novel yet simple steganography scheme. As it will be shown in details in section 4, our analysis indicates that in Standard Arabic the frequency of one diacritic, namely Fatha, is almost equal to the occurrence of the other seven diacritics. Thus, the paradigm we introduce in this work assigns a one bit value, namely 1 value, to the diacritic Fatha and the remaining seven diacritics will represent a value of one bit of 0 value.

To implement this approach, we use a fully diacritized Arabic text as our cover media. A computer program reads the first bit of the embedded data and then compares it with the first diacritic in the cover media. If, for example, the first bit to be embedded was a one, and the first diacritic was a Fatha, the diacritic is kept on the cover media and an index for both of the embedded text and the cover media is incremented. If, however, the first diacritic was not a Fatha then it is removed from the cover media and the index for the cover media is incremented to explore the next diacritic. This approach is repeated until a Fatha is found. The same method is used to implement zeros with the only difference that a zero will search for the other seven diacritics instead of the Fatha. The overall process is repeated for as long as there are bits remaining to be hidden.

The following figures illustrate our proposed technique. Fig. 1 shows a Standard Arabic text full of diacritics. We generate pseudo-random sequences to embed into our cover media, the sequence used in this example is: E7 - 30 - E9 - 1C A4 - FC - B8 - B9 - AF - 1F - 0B - D9 - 22 represented in Hexadecimal format. Finally, Fig. 2 shows how the sequence hidden text can be encoded in the cover media using the presented approach.

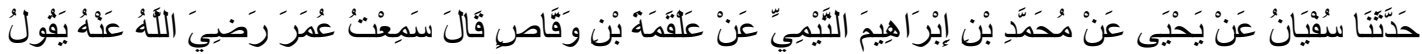

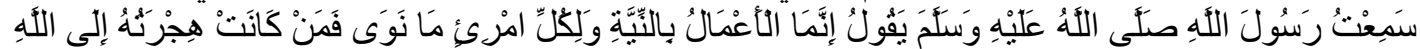

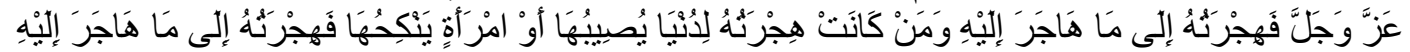

Figure 1. Example of a Standard Arabic text with full diacritics placement.

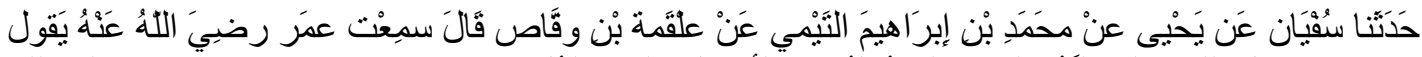

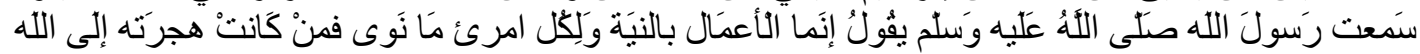

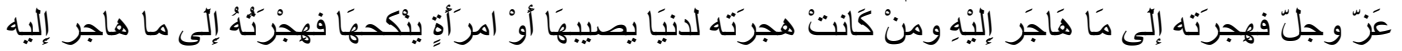

Figure 2. Example of using Standard Arabic diacritics to encode a pseudo-random sequence.

It should be noted that the same cover media can be reused more than once if needed. However, unless a method is used to reinsert removed diacritics, capacity will decrease drastically every time we embed a new message into the text. A research group in IBM [7] proposed to use maximum entropy to restore missing Arabic diacritics. Another approach used HMMs to restore vowels [8]. Diacritic restoration can also be done manually if necessary, and in any case, a new cover media that is already diacritized can be used instead. 


\section{Discussion and Analysis}

This section summarizes the tests ran to evaluate the proposed steganography method, along with some statistics that can enhance this approach. As a testbed, or test environment, we used one of the largest online books available in Arabic literature, namely Musnad Al-Emam Ahmed. Because of its large size, only a portion, i.e. half, of the book was used as a testing environment. The testbed contains 7305490 characters and 1037265 words. Initial results showed very promising expectations as for capacity and ambiguity. Diacritics represent almost 50\% of the total file size if the cover media is fully diacritized. Table 2 shows some statistical studies done on the document.

Having eight different diacritic symbols, we initially assumed that each diacritic symbol (which is a 1 Byte) can carry 3 bits of hidden information at once. This means that we can assume that the usable capacity can be almost $16.7 \%(50 \%$ diacritics multiplied by $3 / 8$ for each Byte). Unfortunately, this is not the case since advanced studies showed huge statistical discrepancies between the different diacritic symbols in the document. It was observed that usually Fatha stands for almost $50 \%$ of the presence of all diacritic marks! Whereas, for example, Tanween Fath only makes up for $0.55 \%$ of the whole set! The previous results indicate that giving each diacritic a set of 3 bits will decrease capacity by a huge rate. For example, if we map 3 to a Fatha and if we map a 1 to a Tanween Fath, we will encounter more than a 100 Fatha's, which we need then to discard, before encountering a one Tanween Fath.

This prompted for a new technique where we assign a one bit value, i.e. 1, to the diacritic Fatha and another bit value, i.e. 0, to any of the other diacritic symbols (i.e. Dhammah, Kasrah ...etc). Mapping each diacritic, which is one byte in size, to a bit of hidden message (1/8) works well, but will reduce the capacity ratio. Theoretically, we can calculate that the best case scenario cannot surpass $6.25 \%(50 \%$ * 1/8). In fact, the average capacity is almost $3.27 \%$ per bit which is half the theoretical rate. On the other hand, this technique is much more ambiguous for general users than the previous technique.

A question arises, is $3 \%$ capacity considered a low ratio in text steganography? Comparing this technique with some other proposed methodologies in the literature, the previous ratio $(3 \%)$ exceeds all the other proposed techniques. The authors in [4] proposed a new approach in Arabic and Persian steganography that used word shifting which produced on average $1.37 \%$ bit per bit. The work in [9] proposed a technique for using typesetting tools and provided a capacity of $0.98 \%$ per character or almost $0.12 \%$ bit per bit. Furthermore, tests showed that the work in [6] introduces an average of $1.22 \%$. Tables 3 and 4 provide more insight to our approach compared to the approach in [6] and in [11].

Some properties of the proposed approach and other remarks can be summarized as following:

- If a fully diacritized Arabic text is used as cover media, the scheme provides the highest capacity.

- The proposed method is robust. It can withstand printing, OCR techniques, font changing, retyping as long as the medium can display Arabic.

- It is fast and does not require large computational power.

- Simple and can be implemented manually if needed.

- Might raise suspicions since it is uncommon nowadays to send diacritized text, unless the cover media used is religious or political documents for example. 
- After embedding the hidden media, some of the words can contain a lot of diacritics on them, while other redundant diacritics can exist depending on the arrangement of diacritics in that word. This can reduce the ambiguity of the technique.

Table 2. Statistical reports for the cover media used as a testbed.

\begin{tabular}{|c|c|c|c|c|}
\hline Diacritics & Fatha & Kasrah & Dhammah & Sukkon \\
Frequency & 1679820 & 472101 & 367224 & 459566 \\
\hline Diacritics & Shaddah & Tanween Fath & Tanween Kasr & Tanween Dham \\
Frequency & 300906 & 18752 & 50820 & 24096 \\
\hline
\end{tabular}

Table 3. Diacritics Technique

\begin{tabular}{|c|c|c|c|}
\hline File Type & File Size (Bytes) & Cover Size (Bytes) & Capacity (\%) \\
\hline .txt & 10,356 & 318,632 & $3.250 \%$ \\
\hline .wav & 43,468 & $1,334,865$ & $3.256 \%$ \\
\hline .jpg & 23,796 & 717,135 & $3.318 \%$ \\
\hline .cpp & 10,356 & 318,216 & $3.254 \%$ \\
\hline & & Average & $3.27 \%$ \\
\hline
\end{tabular}

Table 4. Kashida Technique

\begin{tabular}{|c|c|c|c|}
\hline File Type & File Size (Bytes) & Cover Size (Bytes) & Capacity (\%) \\
\hline .txt & 4439 & 365181 & $1.215 \%$ \\
\hline .html & 4439 & 378589 & $1.172 \%$ \\
\hline .cpp & 10127 & 799577 & $1.266 \%$ \\
\hline .gif & 188 & 15112 & $1.244 \%$ \\
\hline & & Average & $1.22 \%$ \\
\hline
\end{tabular}

\section{Conclusion}

The paper proposed a novel algorithm for text steganography in Arabic language and other similar Semitic languages. This was achieved by taking advantage of the existence of diacritic symbols in Arabic documents. Diacritics were used to represent binary bits depending on the hidden message. It was shown that in a fully diactritized text, capacity can be very high compared to other methods in the literature. The method was implemented and demonstrated satisfactory results.

This method can be used also in printed documents, and can be used with different fonts as well as different machines as long as it supports Arabic language. Different variations of this technique can be implemented and some have been discussed in section 4 .

The presented approach is easy to implement and does not require complex software. A trained human can perform the scheme manually if necessary. In addition, automated tools can be used to reinsert diacritics in the cover media for it to be used again. Further work can be carried out to improve the ambiguity of this method. 


\section{Acknowledgments}

Authors would like to thank King Fahd University of Petroleum \& Minerals (KFUPM), Dhahran, Saudi Arabia, for supporting this research work. Special appreciation to the students of the course COE 509: Applied Cryptosystems Techniques \& Architectures for their valuable initiatives and positive cooperation.

\section{References}

[1] Donovan Artz, "Digital Steganography: Hiding Data within Data," IEEE Internet Computing, vol. 05, no. 3, pp. 75-80, May/Jun, 2001.

[2] Neil F. Johnson and Sushil Jajodia, "Exploring Steganography: Seeing the Unseen," IEEE Computer, February 1998, vol. 31, no. 2, pp.26-34.

[3] J.C. Judge, "Steganography: Past, Present, Future", SANS white paper, November 30, 2001, http://www.sans.org/rr/papers/index.php?id=552, last visited: March 30, 2006.

[4] Shirali-Shahreza, M.H. and Shirali-Shahreza, M., "A New Approach to Persian/Arabic Text Steganography," in th IEEE/ACIS International Conference on Computer and Information Science, 2006. ICIS-COMSAR 2006, Location, 10-12 July 2006, pp. 310-315.

[5] J.T. Brassil, S. Low, N.F. Maxemchuk, and L. O'Gorman, "Electronic Marking and Identification Techniques to Discourage Document Copying", IEEE Journal on Selected Areas in Communications, vol. 13, Issue. 8, October 1995, pp. 1495-1504.

[6] Adnan Gutub and Manal Fattani, "A Novel Arabic Text Steganography Method Using Letter Points and Extensions", WASET International Conference on Computer, Information and Systems Science and Engineering (ICCISSE), Vienna, Austria, May 25-27, 2007.

[7] I. Zitouni, J. S. Sorensen, and R. Sarikaya, "Maximum entropy based restoration of Arabic diacritics." In Proceedings of the 21st international Conference on Computational Linguistics and the 44th Annual Meeting of the ACL Sydney, Australia, pp. 577-584, 2006.

[8] Y. Gal, "An HMM Approach to Vowel Restoration in Arabic and Hebrew." In ACL-02 Workshop on Computational Approaches to Semitic Languages 2002.

[9] Chen Chao, Wang Shuozhong, and Zhang Xinpeng, "Information Hiding in Text Using Typesetting Tools with Stego-Encoding", Proceedings of the First International Conference on Innovative Computing, Information and Control (ICICIC'06),2006.

[10] Farhan Khan and Adnan Gutub, "Message Concealment Techniques using Image based Steganography", The 4th IEEE GCC Conference and Exhibition, Gulf International Convention Centre, Manamah, Bahrain, 11-14 November 2007.

[11] Adnan Gutub, Lahouari Ghouti, Alaaeldin Amin, Talal Alkharobi, and Mohammad K. Ibrahim, "Utilizing Extension Character 'Kashida' With Pointed Letters For Arabic Text Digital Watermarking", International Conference on Security and Cryptography - SECRYPT, Barcelona, Spain, July 28 - 31, 2007. 